\title{
Benign Lymphatic Vessel Neoplasm
}

National Cancer Institute

\section{Source}

National Cancer Institute. Benign Lymphatic Vessel Neoplasm. NCI Thesaurus. Code C6524.

A benign neoplasm arising from the lymphatic vessels. 